\title{
Photospheric fine structure: An observational challenge
}

\section{An analysis of radiative transfer effects on the visibility of small-scale structures}

\begin{abstract}
J. H. M. J. Bruls and O. v. d. Lühe
Kiepenheuer-Institut für Sonnenphysik, Schöneckstr. 6, 79104 Freiburg, Germany

Received 14 August 2000 / Accepted 26 October 2000

Abstract. The requirement on spatial resolution of a large solar telescope is frequently based on the assumption that the smallest observable features in the solar photosphere could not be much different from a photon mean free path due to smoothing by radiative transfer. Although hydrodynamic simulations show that much smaller structures should exist, a resolution of the order of $100 \mathrm{~km}$ is typically considered sufficient for observations in the photosphere. We performed 2D non-LTE radiative transfer computations for thin flux sheets with widths ranging from 10 to $160 \mathrm{~km}$ in the solar photosphere. We demonstrate that such small structures - should they exist could be observed as small scale variations of intensity and polarization. We conclude that the size limit below which photospheric structure cannot be observed due to smoothing radiative transfer effects must lie well below $10 \mathrm{~km}$. A spatial resolution limit for telescopes based on photon mean free path arguments should therefore be abandoned.
\end{abstract}

Key words. line: formation - line: profiles - Sun: photosphere - telescopes

\section{Introduction}

Discussions about the necessary and sufficient angular resolution of a solar telescope often strongly rely on the misconception that structures smaller than a photon mean free path or a pressure scale height are not observable due to the smoothing effect of radiative transfer (in particular scattering). Clearly, this argument is incorrect in an optically thin medium such as the solar corona: we do see a wealth of features that are smaller than the local photon mean free path. In this paper, we will show that it should also be possible to observe solar photospheric fine structure down to a few kilometers in size.

The photon mean free path becomes highly anisotropic in a stratified atmosphere: whereas around monochromatic optical depth $\tau_{\nu}=1$ the horizontal mean free path, $l_{\nu}=1 / \kappa_{\nu} \rho$, is only of the order of $50 \mathrm{~km}$, most photons emitted upwards can escape from the atmosphere $\left(l_{\nu} \rightarrow \infty\right)$. It can therefore only provide an order of magnitude estimate, at best, of the actual interaction distances in the solar atmosphere. Detailed radiative transfer computations are required to figure out whether small features in the atmosphere produce observable intensity variations.

Send offprint requests to: J. Bruls, e-mail: bruls@kis.uni-freiburg.de, http://www.kis.uni-freiburg.de/ ${ }^{\text {bruls }}$
Immediately following the first attempts to formulate and solve the radiative transfer problem in 2 spatial dimensions (Giovanelli 1959; Wilson 1962a,b,c, 1963, 1968), the influence of structuring in the atmosphere has been studied with steadily increasing sophistication, but only in recent years efficient non-LTE methods and radiative transfer codes for 2 or 3 spatial dimensions have become available (Paletou et al. 1993; Auer \& Paletou 1994; Paletou \& Auer 1995; Auer et al. 1994; Trujillo Bueno \& Fabiani Bendicho 1995; Fabiani Bendicho et al. 1997; Rybicki \& Hummer 1991, 1992; Uitenbroek 1999), so that the radiative transfer problem can be solved for multidimensional models with realistic structuring.

Although previous analyses were hampered by lack of computational power, it is worthwhile summarizing some of the results. On the basis of idealized models and restricted non-LTE conditions, most authors concluded on the one hand that if scattering is important, lateral transfer of radiation can play a role over significantly larger distances than a pressure scale height or a photon mean free path, but that on the other hand temperature-sensitive spectral features formed near LTE can be used to resolve small photospheric structures (at least in principle). More specifically:

- Cannon (1976) stresses that a full understanding of multidimensional radiative transfer effects requires 
a high degree of realism in the model, both with respect to the geometry and the physical description of the problem. He warns that common wisdom, obtained from one-dimensional (plane-parallel) radiative transfer computations, may not automatically apply to the multidimensional case. He particularly emphasizes the need for a consistent treatment of photon redistribution, both in angle and in frequency, since the common assumption of complete redistribution for line photon scattering may be totally inadequate in a model with non-negligible velocities. Unfortunately, a consistent treatment of photon redistribution is still a prohibitive requirement in multidimensional non-LTE radiative transfer computations;

- Parameter studies for simplified models, aimed at quantifying the influence of lateral radiative transfer and in particular of scattering, lead to the conclusion that scattering can efficiently equalize the radiation field over horizontal distances significantly larger than a vertical scale height (Stenholm \& Stenflo 1977; Kneer \& Heasley 1979; Kneer 1979, 1981; Mihalas et al. 1978; Owocki \& Auer 1980; Trujillo Bueno \& Kneer 1990);

- Although the CaIIH\&K lines are known to be strongly scattering lines, the fact that the source function is very close to LTE for most of the line wings gives them high diagnostic potential for photospheric small-scale structure (Mihalas et al. 1978; Owocki \& Auer 1980);

- Bright rings are a natural phenomenon when viewing magnetic flux tubes (with a thin boundary layer) in continuum radiation (Caccin \& Severino 1979; Stenholm \& Stenflo 1977; Deinzer et al. 1984; Knölker et al. 1988; Knölker \& Schüssler 1988; Steiner 1994).

The smallest features probably being of magnetic origin, establishing a lower limit to their sizes would require detailed MHD modeling with an accurate treatment of the radiative cooling/heating mechanisms and an extremely high spatial resolution. Among other things, current MHD simulations lack the necessary spatial resolution to reliably determine the size of these features. The main aim of this paper is to show, by means of consistent non-LTE radiative transfer computations, that it is possible, in principle, to directly observe solar photospheric fine structure down to scales of a few kilometers.

We do not touch upon the closely-related issue of deriving information on the atmospheric structuring below the instrumental resolution limit by means of sophisticated line profile inversion techniques, that are now widely used. The first generation of those methods, initially developed to analyze data from the Advanced Stokes Polarimeter (ASP), derives an average 1D Milne-Eddington model atmosphere for each pixel in the image. Later versions allow for multi-component models, hence they explicitly acknowledge the presence of sub-resolution structuring. A slightly different approach is set forward by the MISMA hypothesis (Sánchez Almeida \& Lites 2000, and references therein); instead of postulating and deriving parameters
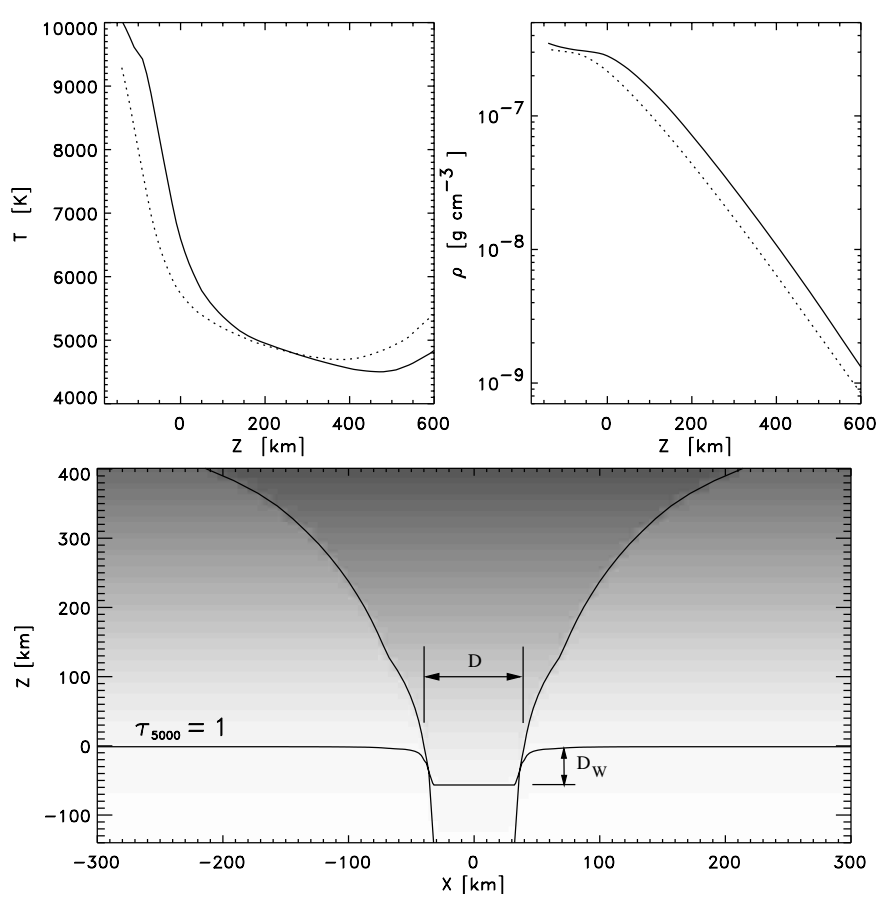

Fig. 1. Top: temperature, $T$, and mass density, $\rho$, for the two components of the sheet models: quiet Sun FAL-C (solid curves) and network flux tube FAL-F (dotted curves). Bottom: geometry of the sheet model; the gray background, which represents $\rho$, clearly distinguishes the sheet from the denser environment. The optical depth unity curve defines the location of $z=0$, at which height the width of the sheet, $D$, and the field strength $B_{0}$ are defined. The Wilson depression, $D_{\mathrm{W}}$, is defined as the height difference between the $\tau_{5000}=1$ levels inside and outside the sheet

for a number of discrete components, it assumes the atmosphere to consist an ensemble of tiny structures (of undefined size) and derives the average and the width of the distribution of the magnetic field and thermodynamic parameters within each resolution element. Although these methods still suffer from various shortcomings - LTE, simple geometry, no radiative interaction between the different components - they provide the precious step beyond the spatial resolution imposed by the instrument, whether that is 1 arcsec (ASP) or 0.025 arcsec (Advanced Solar Telescope operating at diffraction limit).

\section{Models and methods}

\subsection{Atmospheric models}

Since we expect the non-LTE and lateral transfer effects in magnetic flux sheets and tubes to be very similar, we restrict ourselves to the computationally less involved case of a flux sheet, which requires only a $2 \mathrm{D}$ computation. Given that there is quite some uncertainty about the actual size, temperature and density stratification of very small-scale magnetic flux tubes in the solar photosphere, we use standard 1D models for a magnetic network flux tube and the surrounding quiet Sun, FAL-F and FAL-C 


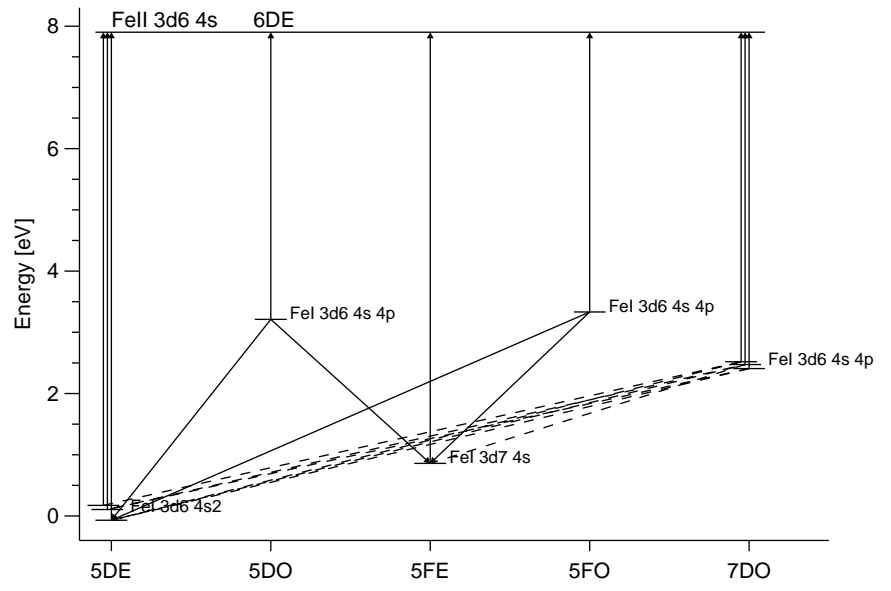

Fig. 2. Termdiagram for the iron atom

(Fontenla et al. 1991), respectively, which are combined into a 2D model through the thin flux-tube approximation (see, e.g., Parker 1974 or Defouw 1976). The geometry of the sheet and the temperature and density of its two components are shown in Fig. 1. After choosing a sheet thickness, $D$, and a magnetic field strength, $B_{0}$, both at height $z=0$ in the quiet-Sun model, all other parameters are automatically fixed. In order to induce only a moderate Wilson depression, $D_{\mathrm{W}}$, we choose $B_{0}=1 \mathrm{kG}$, which is in the lower part of the range given by the observations. The sheet thickness is varied between 10 and $160 \mathrm{~km}$ and the horizontal extent of the computational domain is set to $100 \mathrm{D}$, so that the interference from the periodic horizontal boundary condition is minimal. The vertical extent of the computational domain is chosen so as to comfortably cover the formation heights of the spectral lines and continua considered.

For the purpose of our radiative transfer computations we will assume that magnetic flux sheets may become arbitrarily thin and that they have a sharp boundary, but at the appropriate places we will discuss what the influence of a finite boundary layer would be. Estimates of the thickness of the boundary layer of a typical magnetic flux tube embedded in the quiet solar photosphere are on the order of $10 \mathrm{~km}$ (Schüssler 1986; Knölker et al. 1988), which is much smaller than currently observable.

Our model is static and time-independent, i.e., even though we know that these small structures are very dynamic we will not consider the effects of the rapid radiative heating of the sheet by the environment (Trujillo Bueno \& Kneer 1987; Kneer \& Trujillo Bueno 1987; Fabiani Bendicho et al. 1992) and its impact on the dynamics. The size of the smallest photospheric fine structure may be directly related to the efficiency of the radiative heat exchange with the environment, but on the other hand we will see that even if the temperature of the sheet and the environment are equal at each height the emergent intensities may still be different.

The difference with previous analyses lies with the level of compromises needed to make the problem computationally tractable. We can now combine realistic flux sheet geometries, at arbitrary spatial scales, with consistent non-LTE computations. Still, we are not able to consistently treat photon scattering in case of dynamic models, so that we are essentially restricted to models with very small macroscopic velocities (typically not larger than the thermal velocities of the particles).

Even though our models consist of two onedimensional components, i.e. with only height dependence of the thermodynamic parameters, the resolution of the spatial grid needs careful consideration. In 1D planeparallel radiative transfer computations the spatial grid is in general chosen equidistant in the logarithm of a standard optical depth scale; in the depth range considered here, this corresponds to an approximately equidistant spacing in geometric height, which we will therefore also adopt for the 2D spatial grid. The horizontal resolution is more tricky. On the one hand, we expect rather strong gradients in several parameters near the sheet boundary, which require an increased spatial resolution, and on the other hand, some distance away from the flux sheet everything becomes very smooth, allowing for a reduced resolution. A cartesian grid with typically $145 \times 75$ grid points and a resolution of about $D / 20$ in the central part of the sheet is used.

\subsection{Atomic models}

We perform the non-LTE radiative transfer computations for the FeI 5247 and $5250 \AA$ lines, which are expected to be formed not too far out of LTE.

The iron model atom (Fig. 2) is tailored so that it represents the essential non-LTE effect influencing the formation of these lines, namely the overionization by the superthermal UV radiation field, without becoming overly concerned about the actual accuracy. It consists of only $5 \mathrm{Fe}$ I terms and the Fe II ground state, and their radiative transitions. The choice of the upper terms of Fe I is somewhat arbitrary in the sense that they represent a subset of the terms that contribute to the overionization and have strong radiative transitions to the lower terms. In the (deeper) photosphere, where the neutral iron levels are strongly coupled collisionally as well as radiatively through numerous strong optically thick lines, the overionization results in a collective depression of the populations of all neutral iron levels. As a result, the line opacities significantly deviate from LTE, whereas the line source functions are very close to their LTE values.

The terms are collapsed into a single level and the multiplets are represented by a single line, except where necessary to represent the individual lines. For most lines we use the oscillator strengths, $f$, compiled by Fuhr et al. (1988), which are virtually the same as those determined by Thévenin (1989) by means of LTE line fits to observed quiet-Sun profiles. However, we multiply their values for FeI 5247 and $5250 \AA$ by a factor of 2.5. This adjustment factor, determined by comparing computed quiet-Sun profiles with observed ones, is warranted since we may expect 
the LTE $f$-values to be too small due to the neglect of overionization in above LTE analysis. Nevertheless, this does not automatically imply that the absolute scale of the iron oscillator strengths is actually wrong by a factor of 2.5; more likely, the problem lies with the inadequacy of semi-empirical 1D average quiet-Sun models.

For reasons of limiting the computational effort we use hydrogenic photoionization cross sections, which do not differ markedly from the experimental cross sections listed by Lites (1972). However, they do differ considerably from the most reliable computed cross sections (Bautista 1997), which include numerous resonances and even show an increase towards shorter wavelengths. Fortunately, due to the strong wavelength dependence of the intensities in the solar photosphere, only the part immediately shortward of the bound-free edge is relevant and there the differences between the computed cross sections, with the resonances smoothed, and hydrogenic cross sections are only moderate.

The large uncertainties in the electron collision rates, for which we employ the impact approximation (Seaton 1962), don't play a crucial role in most of the photosphere, where the the population departures of all levels are already kept close together by the radiative coupling. We neglect neutral hydrogen collisions, but note that they would only constrain the population departures yet closer together but not produce essential changes.

The validity of the model atom has been tested by comparing its results for $1 \mathrm{D}$ and $2 \mathrm{D}$ model atmospheres with those obtained for a significantly more complete model atom. We finally note that it is not our intention to exactly model the Fe I 5247 and $5250 \AA$ lines for comparison with observations--which would indeed require the use of the most accurate atomic data available-but to show the behavior of typical photospheric lines in a flux sheet environment.

For the purpose of comparison, we also compute the strongly scattering $\mathrm{Ca} I \mathrm{H} \& \mathrm{~K}$ lines for some of the flux sheet models. We employ the standard 6-level model for calcium (see, e.g., Uitenbroek 1989, and references therein), and also include (angle-averaged) partial redistribution of the line photons. Polarization not being an important diagnostic for these lines, we restrict ourselves to Stokes $I$ profiles for a few inclination angles.

\subsection{The radiative transfer computations}

All non-LTE radiative transfer computations are carried out by means of the new radiative transfer code $\mathrm{RH}$ developed by Uitenbroek (1999) under the assumption that the field-free approximation is valid, i.e. that the magnetic field does not have a noticeable influence on the populations. As shown by Bruls \& Trujillo Bueno (1996) the field-free approximation is a valid assumption for all situations where the equilibrium is not dominated by strong magnetically-sensitive long-wavelength lines.
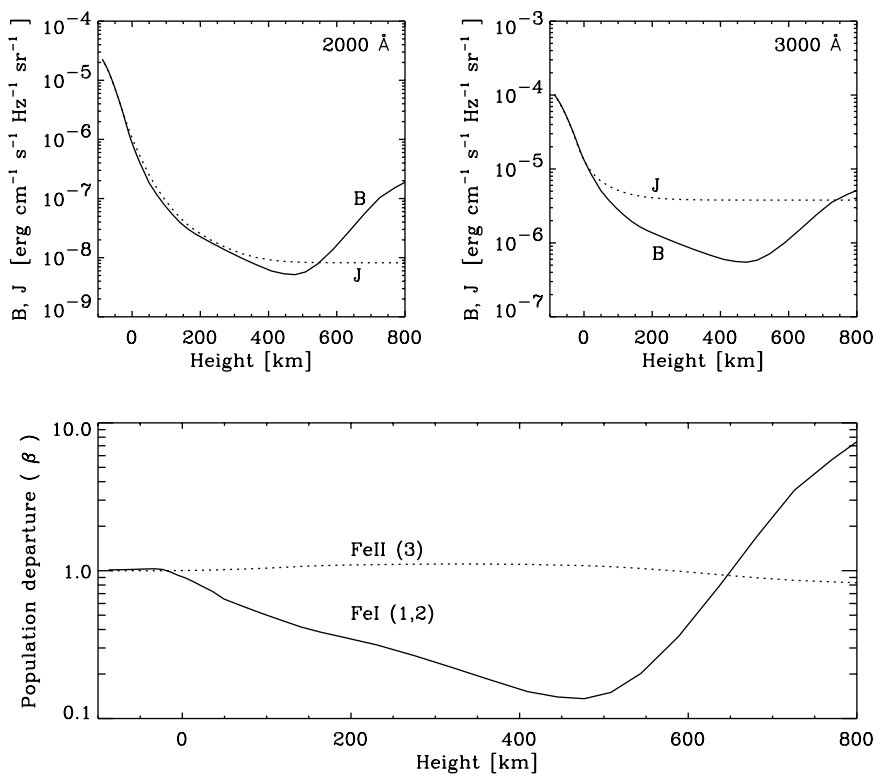

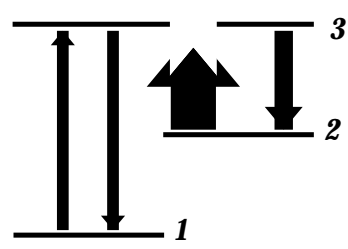

LTE POPULATIONS

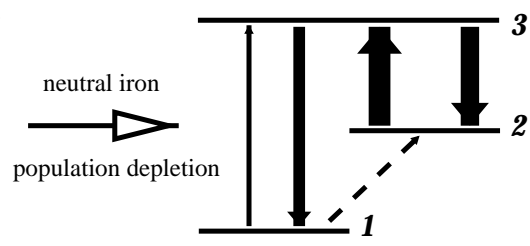

NLTE POPULATIONS
Fig. 3. Properties of the iron ionization balance in the solar photosphere; the 3 levels in the iron schematically represent the low- and mid-excitation neutral levels and the once ionized stage. The ionization equilibrium is set by the ultraviolet radiation field. At wavelenghts shorter than about $2000 \AA$ (upper left), responsible for the photoionization out of the low-excitation levels, $J_{\nu}$ lies close to $B_{\nu}$, whereas at longer wavelengths, contributing to the photoionization out of the mid-excitation levels, $J_{\nu}$ exceeds $B_{\nu}$ (top panels). For LTE populations (bottom left) this means that for level 1 ionization and recombination are in balance (bottom left), whereas for level 2 the photoionization exceeds the recombination. That then leads to depletion of the population of level 2, that is shared with level 1 through strong lines and collisions (middle panel), until there is a balance between the net ionization from level 2 and the net recombination into level 1 (bottom right)

Given the unpolarized non-LTE solution, for the iron lines we also perform a formal solution by means of the SPSR code (Rees et al. 1989; Murphy \& Rees 1990; Murphy 1990) to obtain the Stokes $I$ and $V$ profiles for 8000 equidistant lines of sight in the $x-z$-plane, with inclination $\alpha$ w.r.t. the vertical.

\section{Results: Iron}

\subsection{Statistical equilibrium}

The main deviation from LTE of the iron populations comes from the ionization balance. We therefore discuss that in some detail before continuing with the line profiles. 


\subsubsection{Fe ionization properties for a plane-parallel model}

The formation of Fe I lines has been described in detail by Athay \& Lites (1972), Lites (1972, 1973), and Rutten (1988). The main non-LTE effect on the Fe I photospheric line formation comes from the photoionization. The lowexcitation levels experience an ionizing radiation field that is very close to the Planck function (Fig. 3, upper left), so that photoionization and recombination are virtually in balance. Levels at medium excitation energies, a few electronvolts from the continuum, are ionized by the radiation field near $3000 \AA$; this is superthermal in the photosphere, i.e., the mean (angle-averaged) intensity $J_{\nu}$ exceeds the local Planck function $B_{\nu}(T)$ (Fig. 3, upper right), and the photoionization rate exceeds the recombination rate. As a result, the populations of those levels drop significantly below their equilibrium values, and due to the strong coupling with low-excitation levels (numerous strong lines, collisions) all neutral iron levels have virtually the same population departure coefficient $\beta$ (Fig. 3, middle panel), which is defined as the ratio of the actual population and the corresponding LTE value.

This overall underpopulation reduces the net ionization rate from the mid-excitation levels and induces a balancing net recombination rate into the low-excitation levels (Fig. 3, bottom panel). For our restricted model atom, which lacks the strong lines connecting all the neutral iron levels, there is a small spread of the population departure coefficients, which we may safely ignore: the ground level population departure coefficient, $\beta_{a^{5} \mathrm{D}}$, is a sufficiently accurate proxy for the global iron ionization balance.

\subsubsection{Fe ionization properties for the sheet geometry}

Since the ionization balance is intimately linked to the amount of ionizing UV radiation, its behavior can be explained entirely in terms of the UV radiation field shown in Fig. 4. In case of a pure plane-parallel FAL-C or FAL-F model, we would have found the mean intensity, $J_{\nu}$, to behave as shown in the top right panel of Fig. 3, therefore we will use that as a reference here. That depth-dependence, with appropriate depth-shift to account for the Wilson depression of the sheet, is used to obtain the normalized $J_{\nu}$-values shown in the bottom panel of Fig. 4 . Far from the sheet and at greater heights the $1 \mathrm{D}$ result for $J_{\nu}$ is recovered; deviations occur mainly in the deeper layers of the atmosphere near and inside the sheet. When approaching the sheet, $J_{\nu}$ slightly drops due to the lower level of radiation coming from the direction of the sheet; after crossing the tube boundary, the radiation level is significantly enhanced over the plane-parallel values due to the radiation from the "hot walls" that expose a part of the quiet-Sun atmosphere that is normally hidden. Due to the zero sheet boundary thickness these hot walls are "bare", so that radiation coming from the hot deep photosphere can traverse the entire sheet and even penetrate back into higher layers of the quiet-Sun environment. This
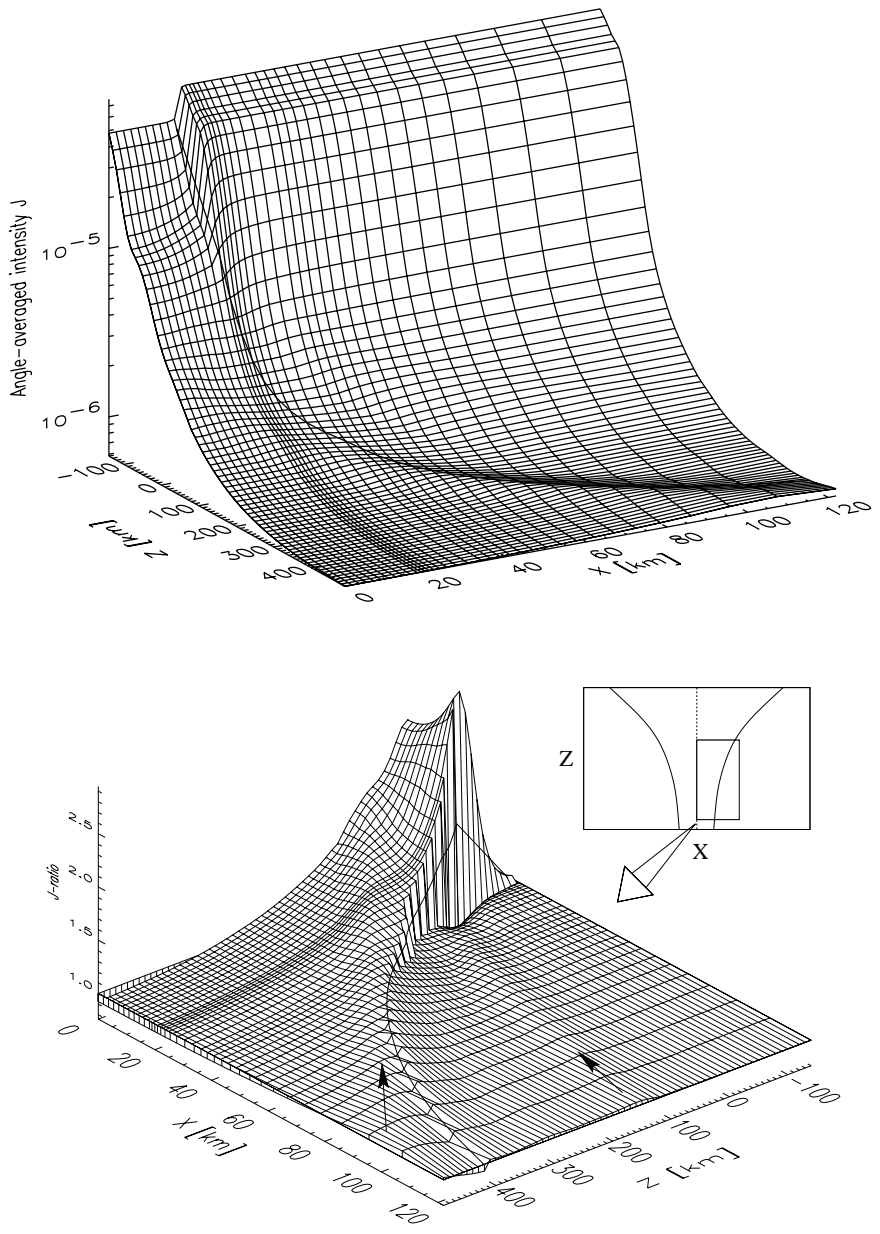

Fig. 4. Angle-averaged intensity, $J_{\nu}$, at $2430 \AA$, respresentative of the entire radiation field that causes the overionization of neutral iron. Top panel: absolute values (erg s${ }^{-1} \mathrm{~cm}^{-2} \mathrm{~Hz}^{-1} \mathrm{sr}^{-1}$ ); bottom panel: $J_{\nu}$ in sheet and environment normalized to the $1 \mathrm{D}$ plane-parallel $J_{\nu}$-values for FAL-F and FAL-C, respectively. The arrows indicate the ripples in the mean intensity that are due to the finite resolution of the angular quadrature used for the integration of the intensities. Note the difference in axis orientation between the two figures, which is necessary to exhibit the behavior near the sheet boundary. As illustrated schematically by the inset, only a small part of the center of the computational domain is shown; the thick curve indicates the location of the sheet boundary

leads to a slight enhancement of $J_{\nu}$ over its plane-parallel value there, as opposed to the region around $z=0$ where $J_{\nu}$ is slightly reduced outside the sheet. Realistic sheet boundary layers would screen the hot walls to some extent, so that the variation of $J_{\nu}$ would be reduced both ways.

It is interesting to note that, although the angular integration of the intensity, required to obtain $J_{\nu}$, is performed by means of a relatively large number of discrete directions - Carlson's (1963) A8 quadrature, with 10 directions per octant, has been used for all computations - in the lower 

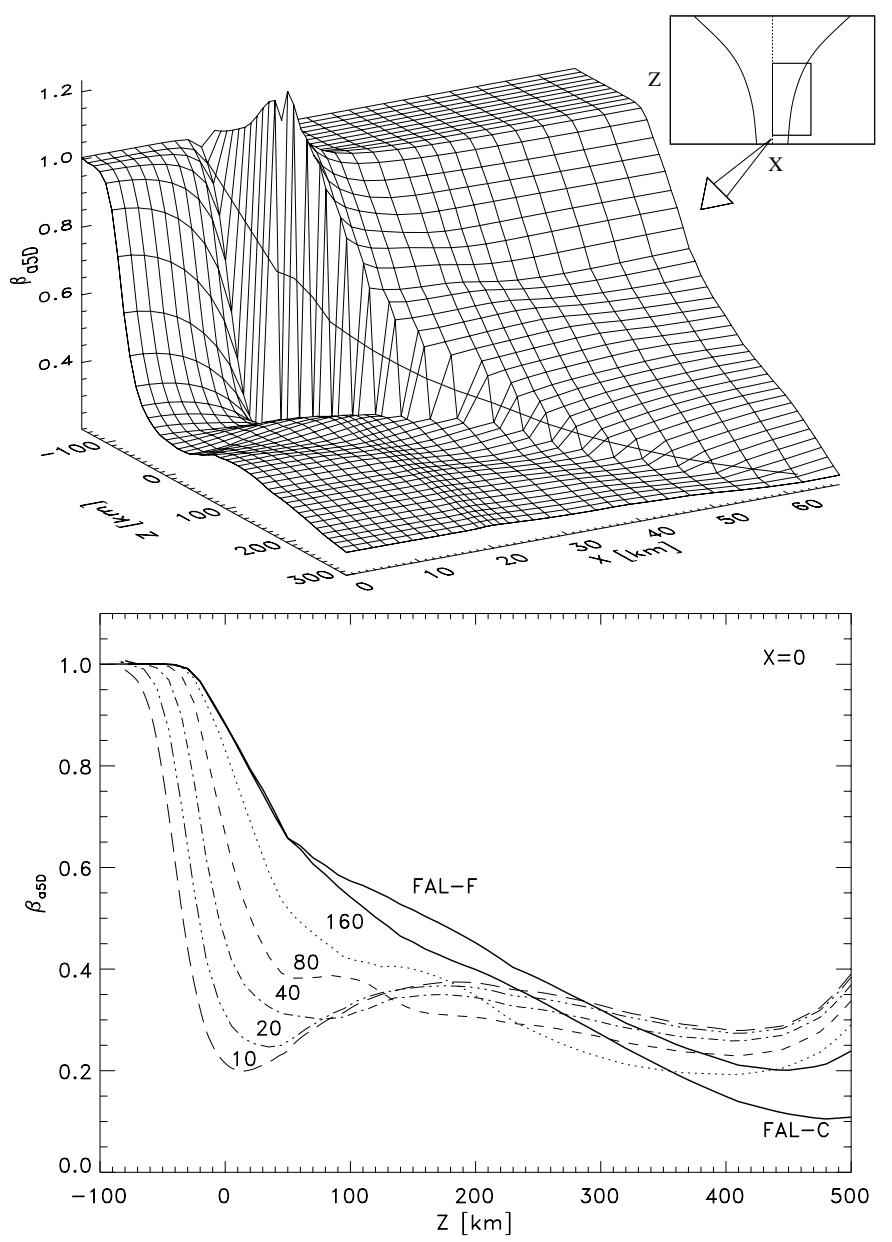

Fig. 5. Iron ionization balance, described by the neutral iron ground level population departure coefficient $\beta_{a^{5} \mathrm{D}}$. Top panel: $\beta_{a^{5} \mathrm{D}}$, as a function of coordinates $x$ and $z$ for the inner parts of the sheet model with $D=40 \mathrm{~km}$. The region shown is somewhat smaller than the one in Fig. 4, and the thick curve again indicates the location of the sheet boundary. Bottom panel: $\beta_{a^{5} \mathrm{D}}$ as a function of height $z$ for all sheet models, labeled by their width $D$, at the sheet center position $x=0$

panel of Fig. 4 we still see three straight ripples (approximately in the direction of the arrows) emerging from the opposite hot wall; the directions correspond to the directions of the angular quadrature. This is a feature typical of $2 \mathrm{D}$ and especially $3 \mathrm{D}$ radiative transfer computations: radiative cooling of hot features occurs preferentially in the directions of the angular quadrature. Fortunately, this effect is negligibly small here, but it can be especially vicious in time-dependent hydrodynamic simulations, where its influence is cumulative and difficult to measure.

The ground level population departure coefficient, $\beta_{a^{5} \mathrm{D}}$, varies roughly as the inverse of the UV radiation field, so that it shows dips where $J_{\nu}$ is enhanced w.r.t. its plane-parallel value and vice versa (Fig. 5). Far from sheet center as well as in the very deep photosphere and at greater heights $\beta_{a^{5} \mathrm{D}}$ does not differ from that in the

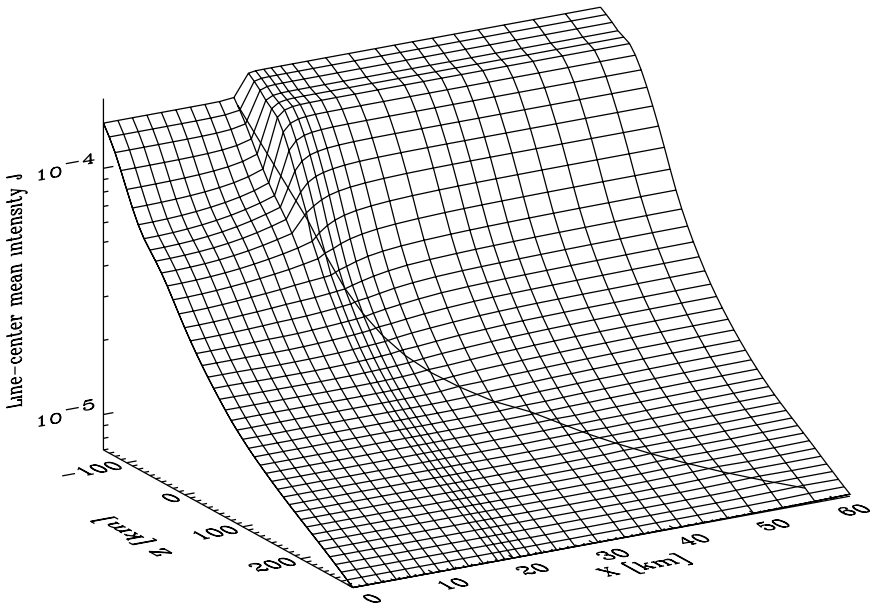

Fig. 6. Angle-averaged intensity at the center of the Fe I $5250 \AA$ line for the sheet model with width $D=40 \mathrm{~km}$. The region shown is the same as in Fig. 5
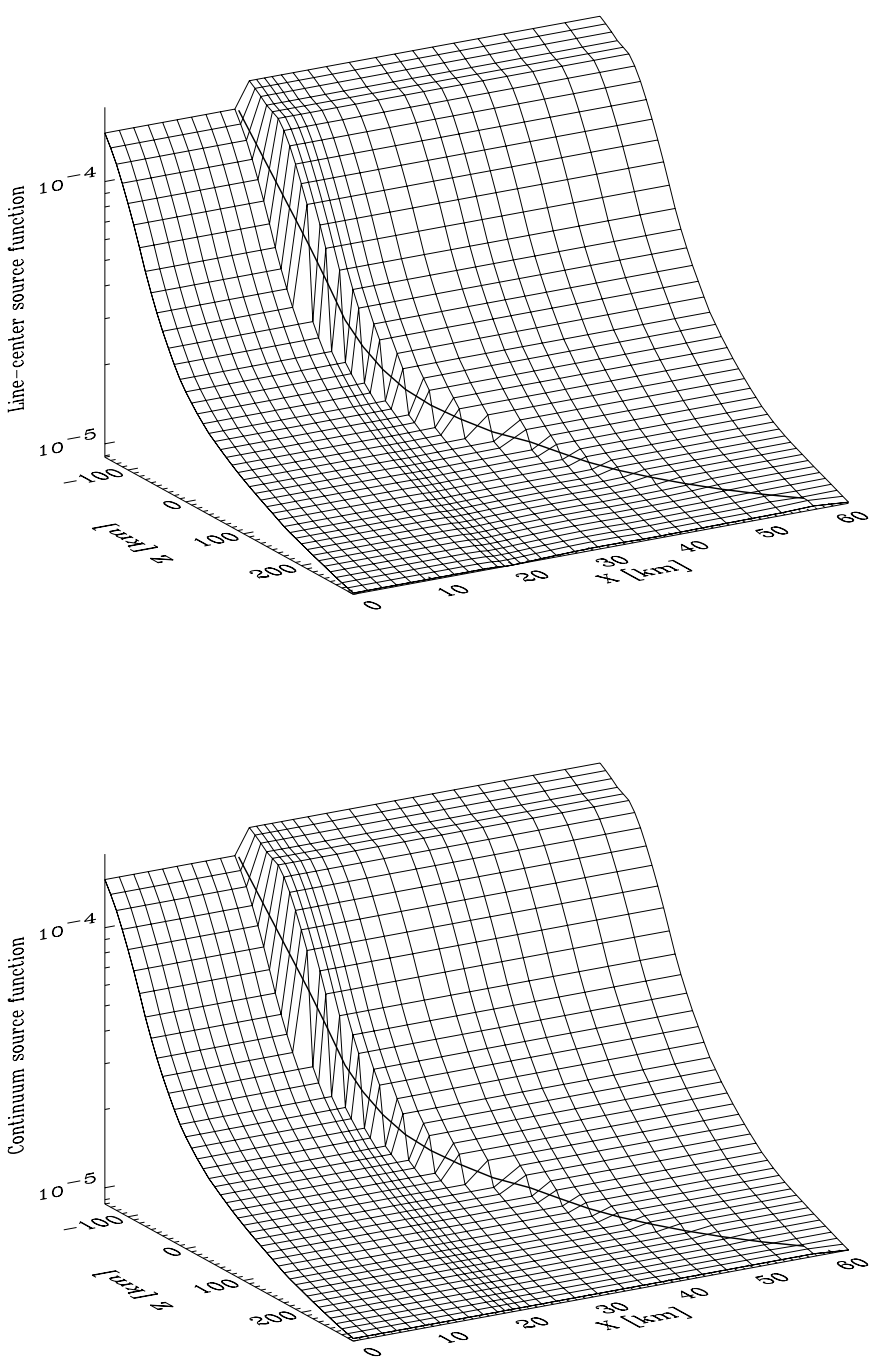

Fig. 7. Source functions for the $5250 \AA$ line center (top), virtually identical to the line source function, and for the neighboring continuum (bottom), for the same region as shown in Fig. 6. Units are the same as for $J_{\nu}$ : $\mathrm{erg} \mathrm{s}^{-1} \mathrm{~cm}^{-2} \mathrm{~Hz}^{-1} \mathrm{sr}^{-1}$ 

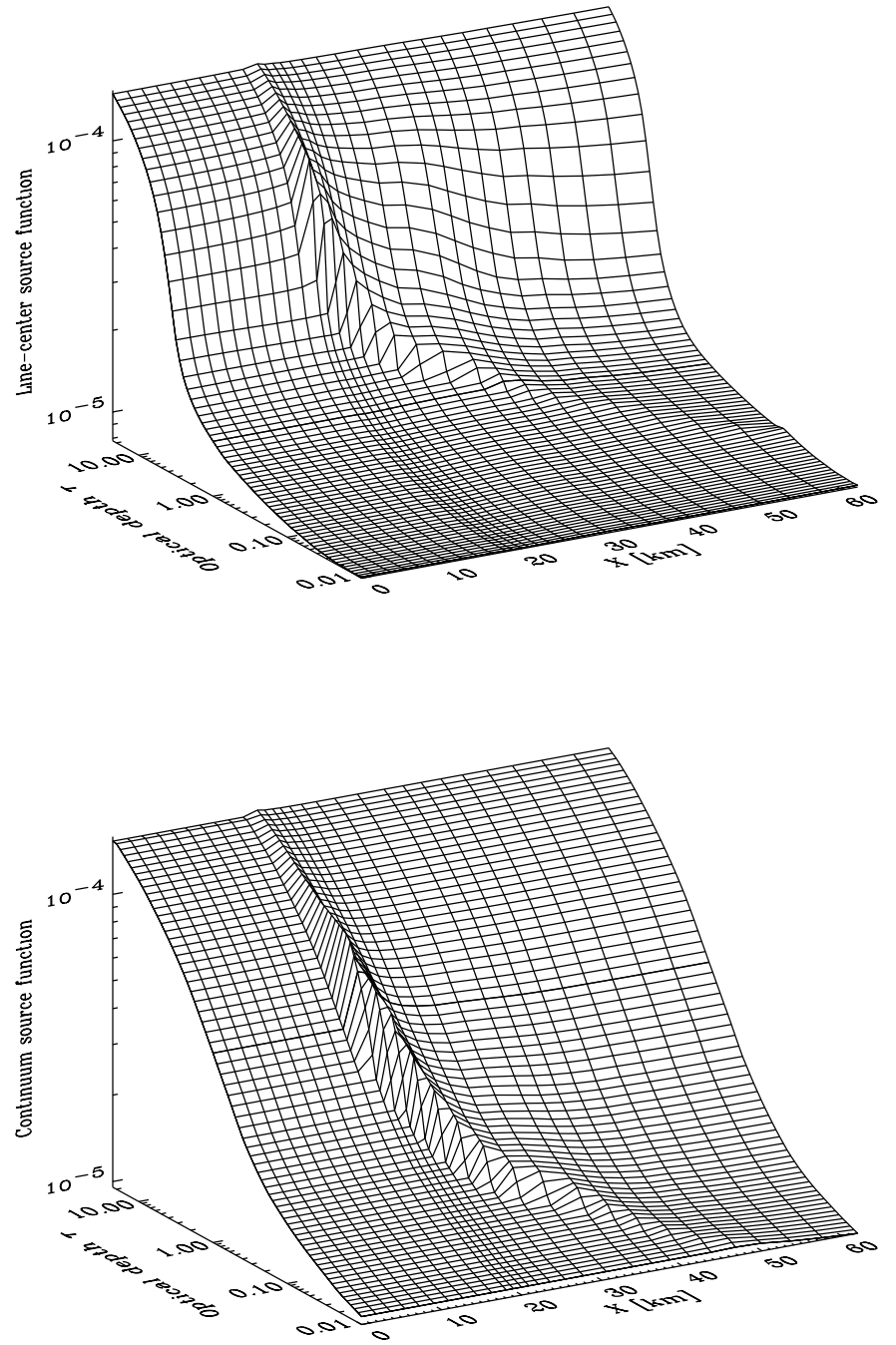

Fig. 8. Same as Fig. 7, but with the height scale replaced by the appropriate monochromatic optical depth scale for each vertical ray. The source functions are nearly homogeneous in this representation, with the exception of a narrow region around the sheet boundary

plane-parallel quiet-Sun model (shown in Fig. 3). As for $J_{\nu}$, the largest deviations from the plane-parallel results occur near height $z=0$, where $\beta_{a^{5}}$ D gradually rises (above unity) when approaching the sheet, then abruptly drops way below unity when crossing the sheet boundary and finally rises again towards the sheet center. The behavior of $\beta_{a^{5} \mathrm{D}}$ as a function of height at the sheet center position, $x=0$, shown in the bottom panel of Fig. 5 , depends on the thickness of the sheet: the thinner sheets suffer significantly from increased overionization as a result of the irradiation by the hot walls, but with increasing sheet thickness $\beta_{a^{5} \mathrm{D}}$ at sheet center approaches its values for the plane-parallel FAL-F model.

Even though the non-LTE effects on the ionization balance are not small, and even though the spatial variation of the ionization balance is not resolved by the spatial resolution chosen for the thicker sheet models, they do not play a decisive role in the present analysis. They merely add to the effects already present due to the density difference between the sheet and its environment.

\subsection{Angle-averaged intensity and source function of the FeI $5250 \AA$ line}

As already shown in Fig. 4 the mean intensity $J_{\nu}$ becomes rather homogeneous in the optically thin upper layers of the atmosphere; scattering may even homogenize $J_{\nu}$ at greater depths. The radiation field in the continuum next to the Fe I $5250 \AA$ line forms no exception, so that we consider only $J_{\nu}$ at line center, which is virtually identical to $\bar{J}=\int \phi_{\nu} J_{\nu} \mathrm{d} \nu$, the mean intensity averaged over the entire line, which constitutes the scattering component of the line source function. We essentially see the same behavior as for the continuum radiation: in the deep photosphere the sheet and the environment are nearly independent of each other - with a jump in $J_{\nu}$ at the sheet boundary - and only above a certain height $(z \approx 0) J_{\nu}$ becomes homogeneous (Fig. 6).

The line source function exhibits similar behavior, except that the height at which it becomes homogeneous lies somewhat higher. This has to with the fact that the $5250 \AA$ line is not a strongly scattering line, so that its source function remains close to the local Planck function until it becomes optically thin; the jump in the line center source function (Fig. 7) reflects the temperature difference across the tube boundary.

For the line formation, it is not the geometrical height scale that plays a role, but the optical depth scale. When plotting the line center and continuum source functions on the appropriate monochromatic optical depth scales, which are different on each vertical ray, a completely new picture emerges (Fig. 8): the Wilson depression is now largely compensated and deviations from homogeneity occur only on a narrow strip along the sheet boundary. The reason for this ridge is clear: on each vertical ray, the transition from the sheet atmosphere into the quiet-Sun environment is accompanied by a significant increase in the opacity; since the opacity itself is an exponential function of depth, already a small distance below the sheet boundary the optical depth will reach the same value as on a ray that runs through the denser quiet-Sun environment all the way from the top of the atmosphere. The source function, however, makes a clean jump at the sheet boundary, so that a different $S(\tau)$ relation results for this transition layer.

\subsection{Line profiles}

Since the emergent intensities do not depend strongly on the sheet thickness, we will discuss the results for $D=40 \mathrm{~km}$ in detail and only indicate the trends when the sheet thickness is varied. Figure 9 displays the Stokes $I$ and $V$ profiles of Fe I $5250 \AA$ for three viewing angles $\alpha$. 


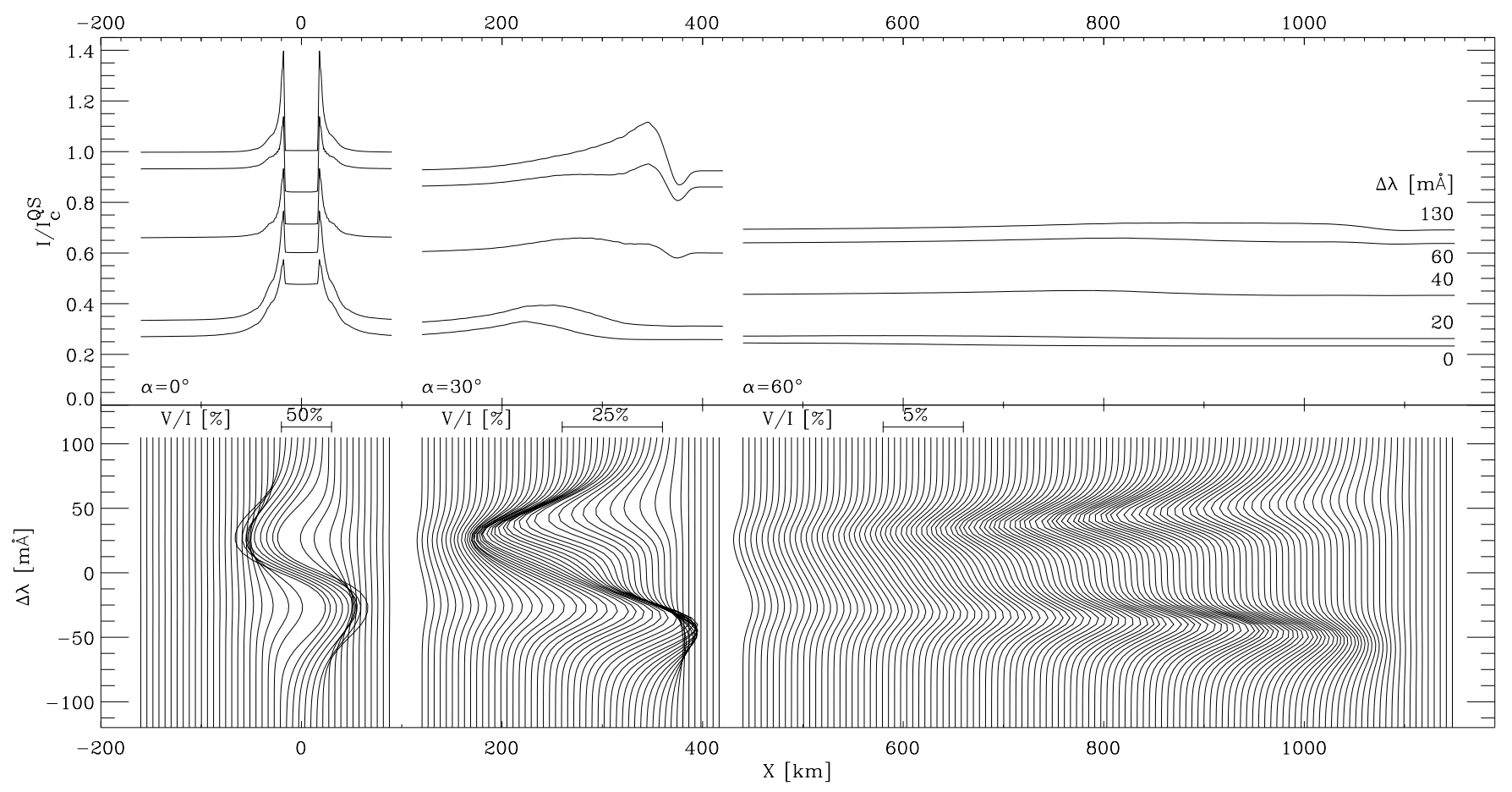

Fig. 9. Stokes $I$ and $V$ profiles for the Fe I $5250 \AA$ line from the $D=40 \mathrm{~km}$ flux sheet at different inclination angles $\alpha$ (from left to right $\alpha=0^{\circ}, 30^{\circ}$ and $60^{\circ}$ ). For non-zero $\alpha, x$ becomes larger because the $x$-axis is fixed where the flux sheet is sitting, at the bottom of the atmosphere, not where it is observed, and the inclination angle $\alpha$ is taken towards positive $x$. For each inclination we select only the region around the corresponding apparent location of the flux sheet. Top: I-profiles, relative to the quiet-Sun disk-center continuum intensity, $I_{\mathrm{c}}^{\mathrm{QS}}$, as a function of the horizontal coordinate $x$ for a few representative values of $\Delta \lambda$, the wavelength relative to line center. Bottom: $V / I$ profiles as a function of $\Delta \lambda$ and $x$; the zero level of each $V / I$ profile, reached at $|\Delta \lambda| \geq 100 \mathrm{~m} \AA$, coincides with the $x$-coordinate of the position (at the surface) for which the profile was computed

For vertical rays (left part of the figure) the hot walls of the sheet are clearly visible as separate entities in the continuum and in the line wings; towards line center the sheet as a whole becomes brighter than its environment. Superposed on the extended intensity enhancement at line center there are two small peaks at the location of the hot walls (near the continuum formation height); these are a result of the sheet boundary being sharp. At all wavelengths, continuum as well as line center, the apparent horizontal extent of the region with enhanced intensities agrees quite well with the actual width of the sheet at the height corresponding to monochromatic optical depth unity.

As soon as the line of sight is inclined by only a small amount, the projection of the height of formation range, typically a few hundred kilometers, exceeds the width of the sheet. In that case the apparent extent of the sheet is no longer a valid measure of its actual thickness. In addition, the amplitude of the intensity enhancements and of the Stokes $V$ profiles decreases quite rapidly with the inclination of the line of sight.

At first sight, part of the Stokes $V$ profiles for the inclined lines of sight $\left(\alpha \neq 0^{\circ}\right)$ look very strange. We know that the Stokes $V$ profiles of the Fe I $5250 \AA$ line should not be completely split at the magnetic field strengths present in our model (about $1 \mathrm{kG}$ ). Nevertheless, some of them appear to be completely split and the separation between the red and blue lobes of Stokes $V$ corresponds to a magnetic field strength of about $1 \mathrm{kG}$. However, this apparent split occurs only on the disk-center side of the sheet, i.e., for those rays that traverse a long path through the nonmagnetic environment before reaching the observer. Near line center the optical thickness of that part of the path may be quite large and as a consequence the polarization signal is lost due to absorption; towards the wings the opacity rapidly decreases and part of the polarization signal is preserved ("ballistic photons"). Neither the width and amplitude nor the separation of the Stokes $V$ lobes contain useful quantitative information, however.

Figure 10 shows an interesting trend in the Stokes $I$ profiles as a function of the sheet width $D$; this has important observational consequences. Consider the results for the different inclinations of the lines of sight separately.

- At disk center (line of sight inclination $\alpha=0$ ) only the hot walls stand out in the continuum radiation. The intensity enhancement, about $40 \%$, is determined mainly by the opacity jump at the sheet boundary; it depends on $D$ only indirectly, e.g., through the variation of the ground level population departures. The width of the region with enhanced intensity is approximately linear with $D$, since it reflects the variation of the sheet thickness with height over the formation 


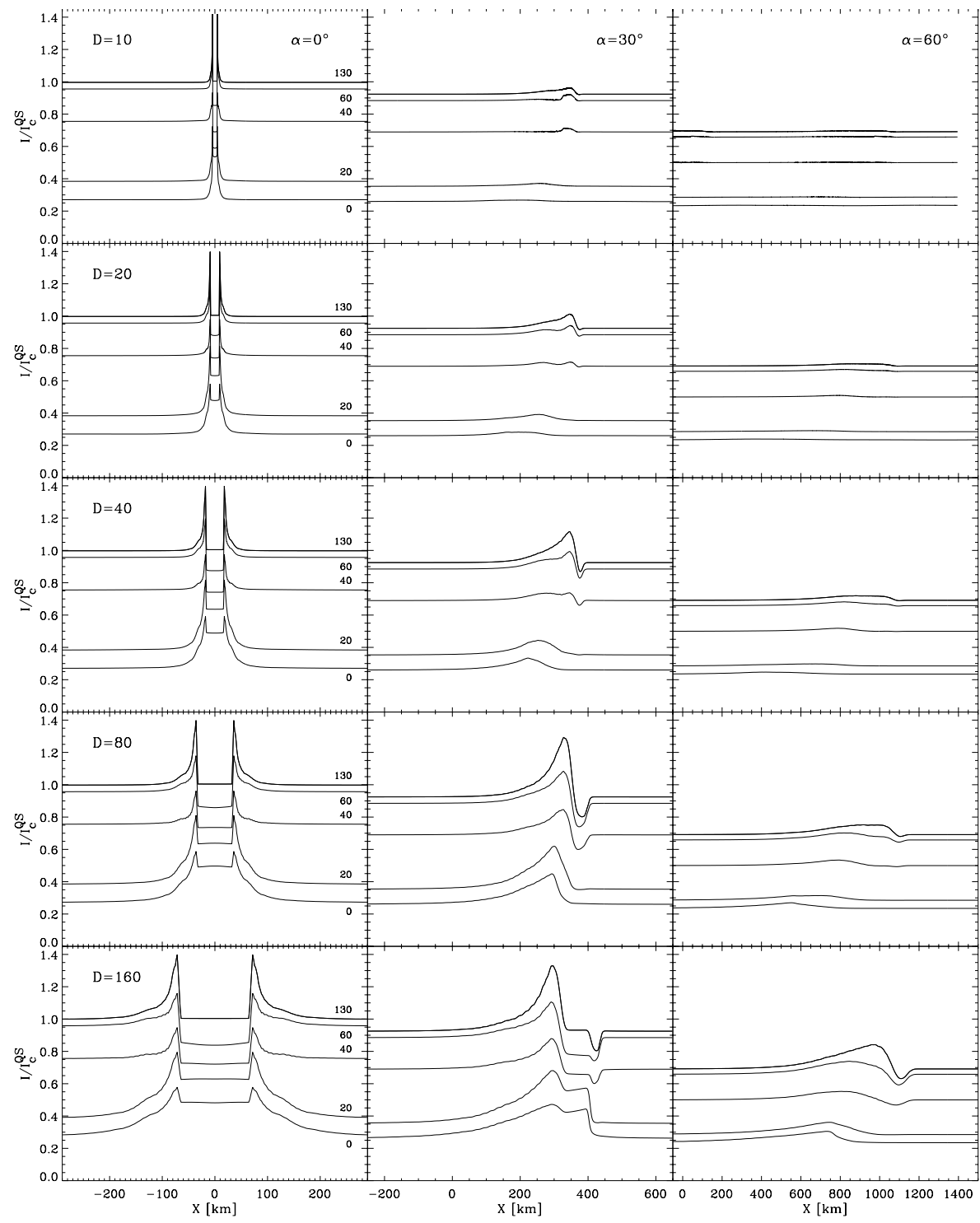

Fig. 10. Stokes $I$, normalized to the quiet-Sun disk-center continuum intensity, $I_{\mathrm{c}}^{\mathrm{QS}}$, as a function of the horizontal coordinate $x$, at the wavelengths $\Delta \lambda$ indicated next to the profiles in the left column (in $\mathrm{m} \AA$ relative to line center, as in Fig. 9). Left to right: line of sight inclinations $\alpha=0^{\circ}, 30^{\circ}$, and $60^{\circ}$ ); top to bottom: sheet widths $D=$ $10,20,40,80$, and $160 \mathrm{~km}$ range of the radiation. For the thicker sheets, these intensity enhancements should be observable with current telescopes, although significantly smeared out and with reduced contrast. The true width of the intensity peaks associated with the walls will only be visible at very high spatial resolution (0.01 arcsec). Towards line center, the entire sheet becomes bright and its observability improves drastically;

- For inclined lines of sight several effects influence the observability of a flux sheet.

- The extent of the region with enhanced intensity grows slightly with $D$;

- The dependence of the intensity enhancement on the limb distance $\mu=\cos \alpha$ depends on $D$ very strongly due to geometry effects: the thinner sheets become invisible at larger $\alpha$, whereas the thicker sheets become brighter than the environment with increasing $\alpha$;

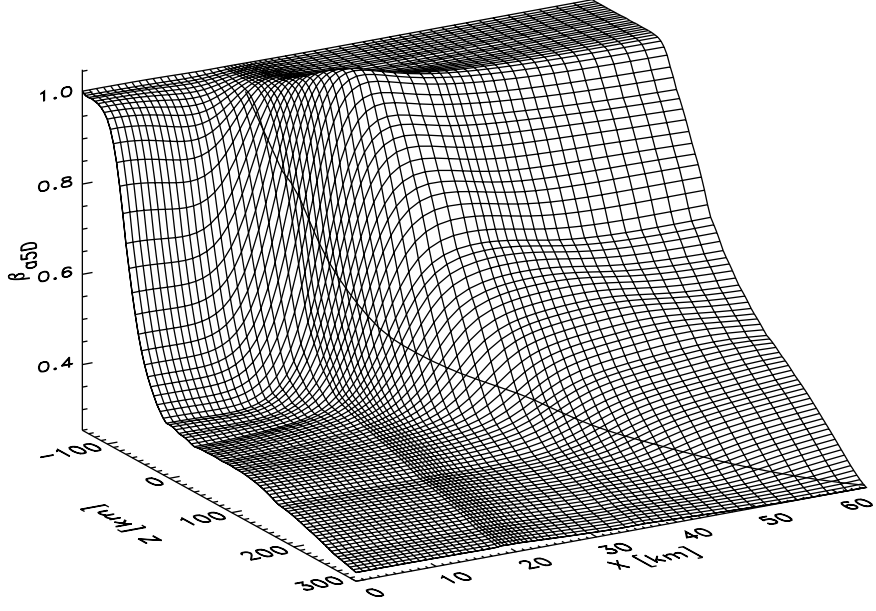

Fig. 11. Neutral iron ground level population departure coefficient $\beta_{a^{5} \mathrm{D}}$ as a function of $x$ and $z$ for the inner parts of the sheet model with $D=40 \mathrm{~km}$ and a $10 \mathrm{~km}$ boundary layer 


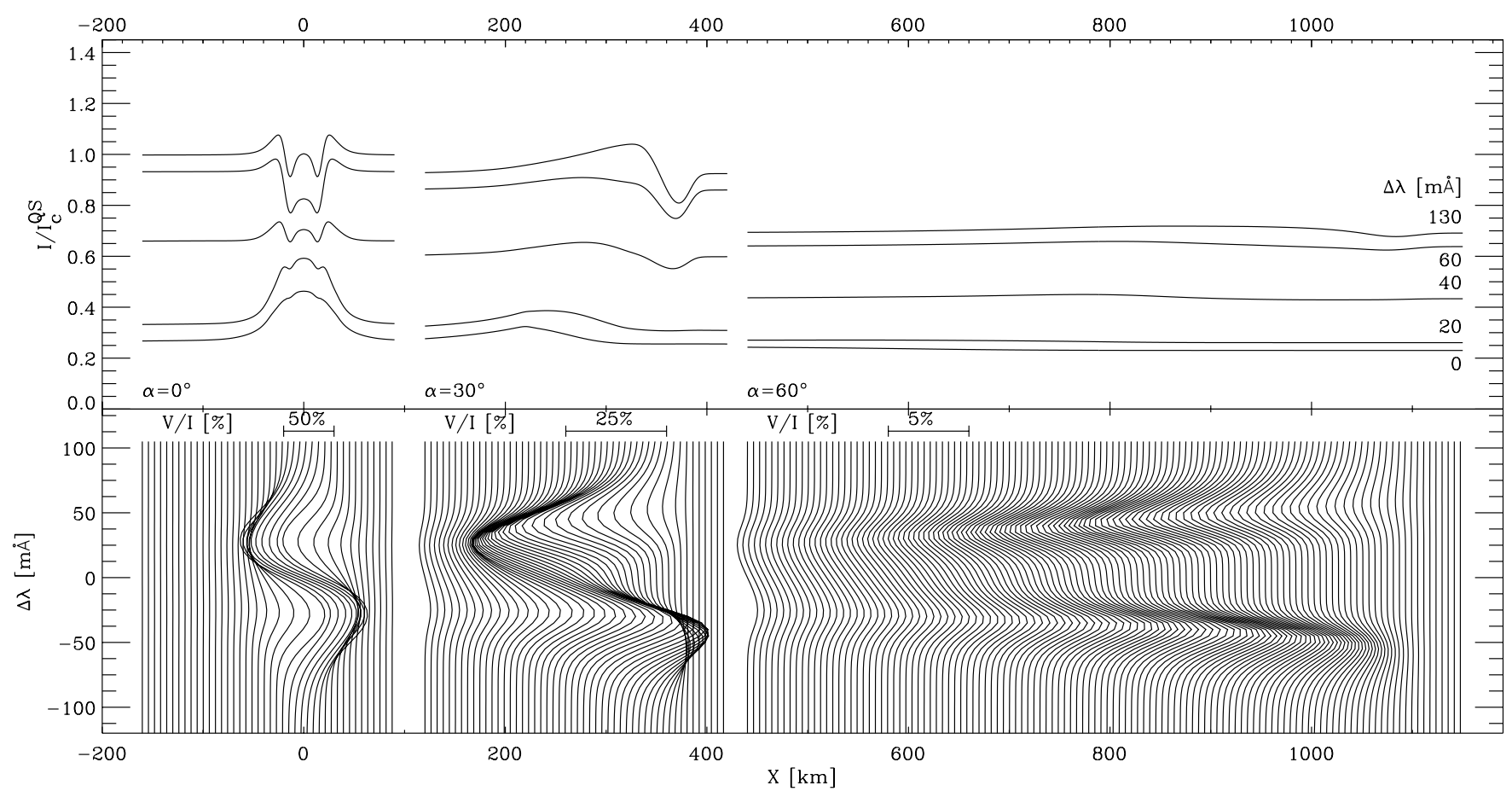

Fig. 12. Same as Fig. 9, but for a sheet model with a smooth $10 \mathrm{~km}$ thick boundary layer

- At given $\mu$, the intensity enhancement itself increases with $D$.

This means that away from disk center the observability of flux sheets rapidly improves with increasing thickness. In the line core the sheet appears as an extended bright region and in the continuum it appears as a combination of an extended bright region and a smaller darker region at the disk-center side.

These findings do not contradict observations at current spatial resolution levels: network and plage regions only seem to have clear excess intensity towards the limb, but they are not visible at disk-center except in the cores of very strong lines (e.g., Ca II H \& K), which are of chromospheric origin, and possibly as $G$-band bright points. At higher spatial resolution, however, even the smallest diskcenter features should be observable in the continuum as well as in cores of many lines.

\subsection{Influence of a smooth sheet boundary}

In order to obtain an impression of the effects of a smooth sheet boundary, we also ran a simulation for the $D=$ $40 \mathrm{~km}$ model with a smooth (tanh-like) $10 \mathrm{~km}$ thick transition between the sheet interior and the ambient quiet Sun. This simulation uses a finer spatial grid, so that the transition layer is resolved well. Such a model results in a smoother behavior of the ground level population departure (Fig. 11) and somewhat less overionization of neutral iron at lower photospheric levels inside the sheet. Part of the UV radiation from the quiet-Sun environment that is responsible for the jump in $\beta_{a^{5} \mathrm{D}}$ at the sheet boundary (Fig. 5) is now absorbed in the transition layer. As a result of that layer, there is no longer a jump in the opacity (at any wavelength) when going from the sheet interior into the quiet-Sun environment, so that the corresponding optical depth scale $\tau_{\nu}^{\text {smooth }}(z)$ also varies more smoothly than the optical depth scale $\tau_{\nu}^{\text {jump }}(z)$ in the model with sharp walls. Concretely, due to the very non-linear relation between opacity and temperature, for a given vertical ray $\tau_{\nu}^{\text {smooth }}(z)$ is initially the same as $\tau_{\nu}^{\text {jump }}(z)$, but just above the sheet boundary $\tau_{\nu}^{\text {smooth }}(z)$ will start to exceed $\tau_{\nu}^{\text {jump }}(z)$. After crossing the sheet boundary into the environment, $\tau_{\nu}^{\text {jump }}(z)$ will increase faster with depth and eventually it will exceed $\tau_{\nu}^{\text {smooth }}(z)$. This in turn will modify the $S_{\nu}\left(\tau_{\nu}\right)$ relation throughout the transition layer and in particular it will reduce the emergent intensity in the outer parts of the sheet.

The resulting line profiles are shown in Fig. 12 (cf. Fig. 9). As expected, only the profiles at disk center are affected significantly: the intensity enhancement due to the hot walls is reduced considerably, and at line center the little peaks originating directly from the hot walls have disappeared. The dips in the continuum and line wing intensity just inside the sheet boundary may come as a surprise, but they are a direct consequence of the modified $S_{\nu}\left(\tau_{\nu}\right)$ relation: on rays just inside the sheet boundary the higher temperature and source functions resulting from the smoothing of the transition layer are more than compensated by the very rapid increase of the optical depth, so that near optical depth unity the source function is actually smaller than in the model with sharp sheet boundary. A sheet with a smooth boundary layer is therefore more difficult to observe, especially in the continuum. 

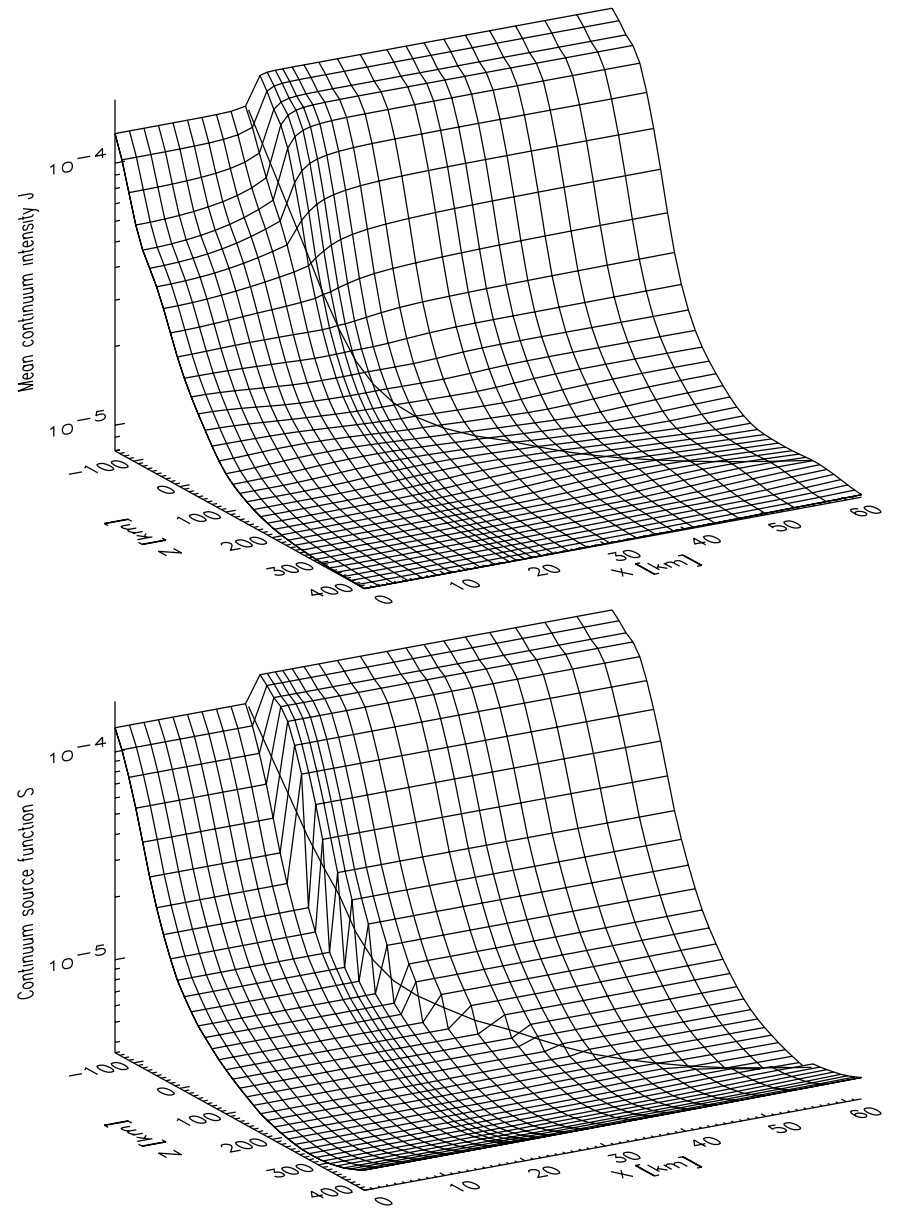

Fig. 13. Angle-averaged intensity (top) and source function (bottom) for the continuum near the Ca II $\mathrm{H}$ line for the sheet model with width $D=40 \mathrm{~km}$

\section{Results: Calcium}

The ionization state of calcium in the solar photosphere and chromosphere needs no discussion: the once ionized state completely dominates throughout the entire height range considered, so that deviations from the equilibrium ionization state are irrelevant. Complications arise from the strongly scattering $\mathrm{H} \& \mathrm{~K}$ resonance lines which essentially dictate the excitation balance in our model atom in a 2-level atom fashion. Due to the high formation of the lines and their strongly scattering character, partial frequency redistribution of line photons needs to accounted for in our computations.

\subsection{Angle-averaged intensity and source function}

The angle-averaged intensity $J_{\nu}$ and the source function $S_{\nu}$ are dominated by the scattering processes in the $\mathrm{H} \& \mathrm{~K}$ lines, which tend to smooth any signature arising from small features in the upper photosphere and chromosphere, where the central parts of these lines are formed. However, in the photosphere the small mean free path
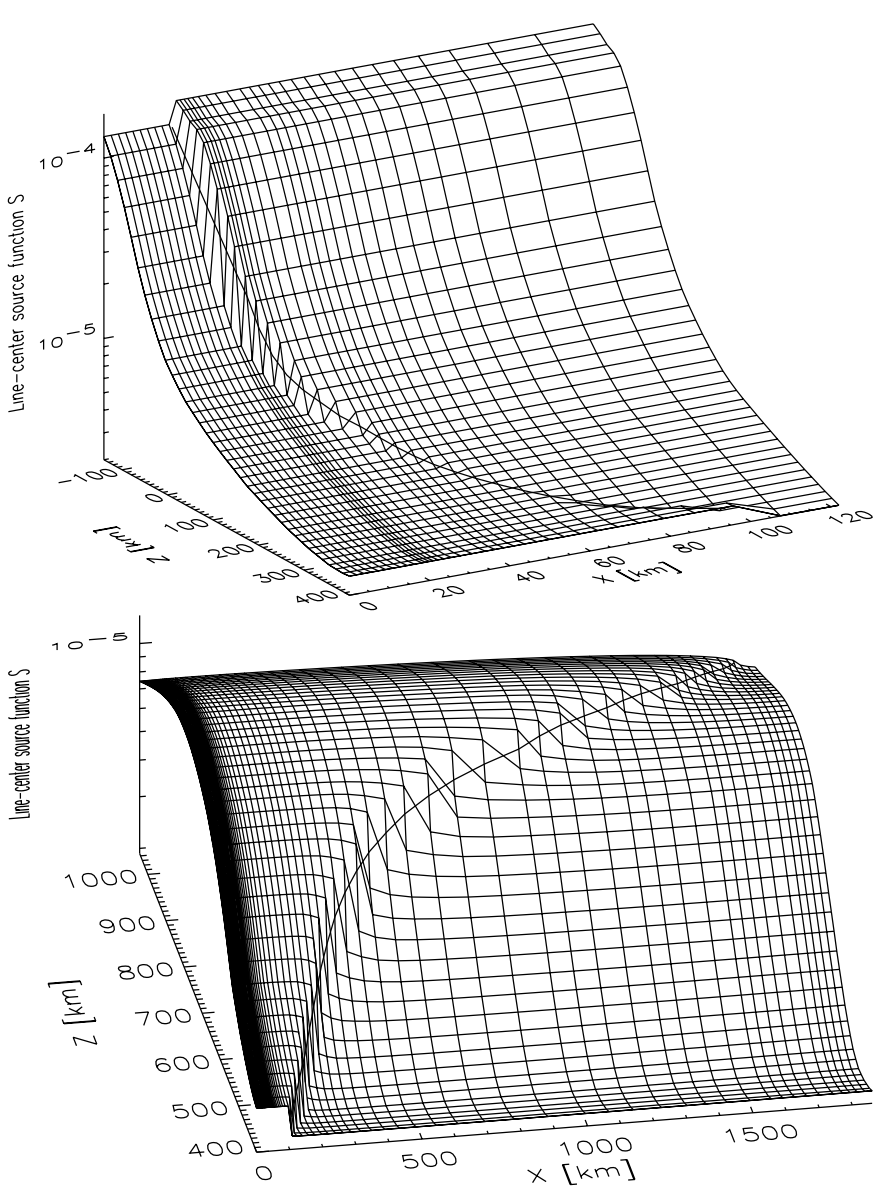

Fig. 14. CaII $H$ line-center source function $S_{\nu_{0}}$ for the sheet model with width $D=40 \mathrm{~km}$. The top panel shows the photospheric part, where the sheet is very thin, and the bottom panel shows the extended-width sheet above the temperature minimum with reversed contrast. Near the flux sheet merging height, about $1000 \mathrm{~km}$, the line starts to feel the outer atmosphere and $S_{\nu_{0}}$ decreases again

length of the line photons (of the order of a meter) keeps the line source function very close to its LTE value, so that the spatial variation of the temperature is reflected in the emergent intensities in the continuum and the line wings.

At continuum and line wing wavelengths $J_{\nu}$ has a smooth transition between the sheet and the environment in the deeper photosphere and it becomes homogeneous above about $z=0$, whereas the source function follows the local Planck function more closely (Fig. 13). At line center, where the source function is equal to the mean intensity, a clear distinction between the sheet and the environment remains up to the flux sheet merging height (about $z=1000 \mathrm{~km}$, Fig. 14). And even at that height the line core is still very optically thick, so that little or no spatial intensity variation is expected in the line core.

Note, that since $S_{\nu_{0}}$ closely follows the local Planck function as long as the line is sufficiently optically thick, the contrast between sheet and environment changes sign 


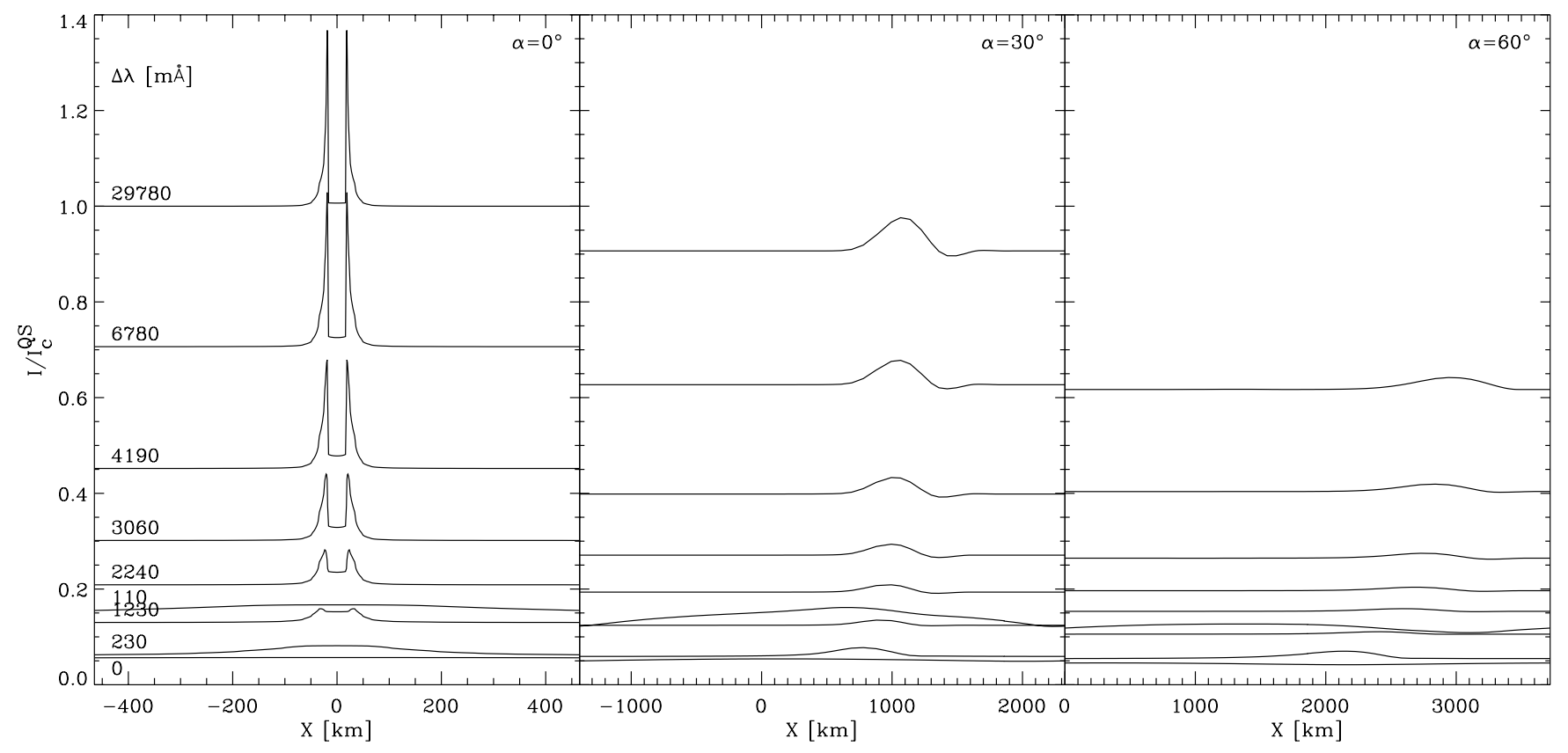

Fig. 15. Intensities, at selected wavelengths $\Delta \lambda$ in the Ca II H line, as a function of horizontal coordinate $x$, for the sheet with $D=40 \mathrm{~km}$ and line of sight inclinations $\alpha=0^{\circ}, 30^{\circ}$ and $60^{\circ}$ (left to right). Note that $\Delta \lambda=110$ and $230 \mathrm{~m} \AA$ correspond to the $H_{2 \mathrm{R}}$ peak and the $H_{1 \mathrm{R}}$ minimum, respectively

near the temperature minimum: in the photosphere we have $S_{\nu_{0}}^{\text {sheet }}<S_{\nu_{0}}^{\text {environment }}$ (Fig. 14, top panel) and higher up that is reversed (bottom panel).

\subsection{Line profiles}

Figure 15 displays the emergent intensities for the $D=$ $40 \mathrm{~km}$ model for a set of wavelengths covering relevant parts of the CaII $\mathrm{H}$ line. The behavior is not principally different from that of the Fe $5250 \AA$ line, except at the very core of the line which is formed high in the chromosphere where scattering plays an important role in smearing out smaller features. At disk center, the hot walls are again clearly visible as separate entities in the continuum and in the outer line wings, the purely photospheric parts of the line; in the inner wings the sheet as a whole becomes brighter. Even closer to line center, at the $H_{1 \mathrm{R}}$ minimum $(\Delta \lambda=230 \mathrm{~m} \AA)$ and the $H_{2 \mathrm{R}}$ peak $(\Delta \lambda=110 \mathrm{~m} \AA)$, which are formed near and above the temperature minimum, the extent of the area with enhanced intensity rapidly grows due to the expansion of the flux sheet with height, but the intensity enhancement itself decreases. At line center, finally, the flux sheet is invisible.

Away from disk center the photospheric parts of the line behave the same as the Fe I $5250 \AA$ line, and towards line center the strong scattering in the chromosphere again erases any traces of enhanced intensities.

\section{Conclusion}

Based on our non-LTE computations for schematic, but not too unrealistic, flux sheet models we come to the following conclusions.

- There is no such thing as a universal size limit below which a photospheric structure cannot be observed due to smoothing radiative transfer effects. The actual observation limit strongly depends on viewing angle and wavelength, geometry of the model and parameters like temperature and density contrasts and gradients;

- Even the smallest features considered in this analysis are still clearly visible, at least from certain viewing angles and at well-chosen wavelengths, so that we expect the observation limit for this kind of structures to lie (well) below $10 \mathrm{~km}$. It seems unlikely, however, that the kilometer-sized microstructures put forward in the MISMA hypothesis can be observed directly;

- As demonstrated with the Ca II H-line, strong scattering alone is not enough to erase all small-scale structure. In the photosphere, where the mean free path of line photons is extremely small, scattering is incapable of smoothing the radiation field; the sheet is therefore visible throughout most of the line.

- At chromospheric heights, due to the larger photon mean free path lengths scattering plays an important role in smoothing the angle-averaged radiation field, but it does not necessarily destroy all spatial intensity 
variation. A thin flux sheet can still be observed, be it as spatially extended features, at the $\mathrm{CaII} \mathrm{H}_{2}$ peaks, even though these are formed in the chromosphere.

Acknowledgements. In this analysis we made use of computer codes provided by Drs. S. Solanki (THIN/LOS for generating the $2 \mathrm{D}$ thin flux tube model atmospheres), H. Uitenbroek (RH for the 2D non-LTE radiative transfer computations) and $G$. Murphy (SPSR for the formal Stokes profile solution).

\section{References}

Athay, R. G., \& Lites, B. W. 1972, ApJ, 176, 809

Auer, L. H., Fabiani Bendicho, P., \& Trujillo Bueno, J. 1994, A\&A, 292, 599

Auer, L. H., \& Paletou, F. 1994, A\&A, 284, 675

Bautista, M. A. 1997, A\&AS, 122, 167

Bruls, J. H. M. J., \& Trujillo Bueno, J. 1996, Sol. Phys., 164, 155

Caccin, B., \& Severino, G. 1979, ApJ, 232, 297

Cannon, C. J. 1976, A\&A, 52, 337

Carlson, B. G. 1963, in Methods in Computational Physics, vol. 1, ed. B. Alder, \& S. Fernbach, 1

Defouw, R. J. 1976, ApJ, 209, 266

Deinzer, W., Hensler, G., Schüssler, M., \& Weisshaar, E. 1984, A\&A, 139, 435

Fabiani Bendicho, P., Kneer, F., \& Trujillo Bueno, J. 1992, A\&A, 264, 229

Fabiani Bendicho, P., Trujillo Bueno, J., \& Auer, L. 1997, A\&A, 324, 161

Fontenla, J. M., Avrett, E. H., \& Loeser, R. 1991, ApJ, 377, 712

Fuhr, J. R., Martin, G. A., \& Wiese, W. L. 1988, J. Phys. Chem. Ref. Data, 17, Suppl. 4

Giovanelli, R. G. 1959, Australian J. Phys., 12, 164

Kneer, F. 1979, Osservazioni e Memorie dell' Osservatorio Astrofisico di Arcetri, 106, Proceedings of the JOSO Workshop "Future solar optical observations needs and constraints", 204

Kneer, F. 1981, A\&A, 93, 387

Kneer, F. \& Heasley, J. N. 1979, A\&A, 79, 14

Kneer, F., \& Trujillo Bueno, J. 1987, A\&A, 183, 91

Knölker, M., \& Schüssler, M. 1988, A\&A, 202, 275

Knölker, M., Schüssler, M., \& Weisshaar, E. 1988, A\&A, 194, 257

Lites, B. W. 1972, Observation and Analysis of the Solar
Neutral Iron Spectrum, High Altitude Observatory, Boulder: NCAR Cooperative Thesis, No. 28

Lites, B. W. 1973, Sol. Phys., 32, 283

Mihalas, D., Auer, L. H., \& Mihalas, B. R. 1978, ApJ, 220, 1001

Murphy, G. A. 1990, The Synthesis and Inversion of Stokes Spectral Profiles, High Altitude Observatory, Boulder: NCAR Cooperative Thesis, No. 124

Murphy, G. A., \& Rees, D. E. 1990, Operation of the Stokes Profile Synthesis Routine, High Altitude Observatory, Boulder: NCAR Technical Note NCAR/TN-348+IA

Owocki, S. P., \& Auer, L. H. 1980, ApJ, 241, 448

Paletou, F., \& Auer, L. H. 1995, A\&A, 297, 771

Paletou, F., Vial, J. C., \& Auer, L. H. 1993, A\&A, 274, 571

Parker, E. N. 1974, ApJ, 190, 429

Rees, D. E., Murphy, G. A., \& Durrant, C. J. 1989, ApJ, 339, 1093

Rutten, R. J. 1988, in Physics of Formation of FeII Lines Outside LTE, ed. R. Viotti, A. Vittone, \& M. Friedjung, IAU Colloquium 94 (Dordrecht: Reidel), 185

Rybicki, G. B., \& Hummer, D. G. 1991, A\&A, 245, 171

Rybicki, G. B., \& Hummer, D. G. 1992, A\&A, 262, 209

Sánchez Almeida, J., \& Lites, B. W. 2000, ApJ, 532, 1215

Schüssler, M. 1986, in Small Scale Magnetic Flux Concentrations in the Solar Photosphere, ed. W. Deinzer, M. Knölker, \& H. H. Voigt, Abhandl. Akad. Wiss. Göttingen, Math.-Phys. Klasse Dritte Folge, No. 38 (Göttingen: Vandenhoeck und Ruprecht), 103

Seaton, M. J. 1962, Proc. Phys. Soc. London, 79, 1105

Steiner, O. 1994, in Infrared Solar Physics, ed. D. M. R. et al., IAU, 407

Stenholm, L. G., \& Stenflo, J. O. 1977, A\&A, 58, 273

Thévenin, F. 1989, A\&AS, 77, 137

Trujillo Bueno, J., \& Fabiani Bendicho, P. 1995, ApJ, 455, 646

Trujillo Bueno, J. \& Kneer, F. 1987, A\&A, 174, 183

Trujillo Bueno, J. \& Kneer, F. 1990, A\&A, 232, 135

Uitenbroek, H. 1989, A\&A, 213, 360

Uitenbroek, H. 1999, in High Resolution Solar Physics: Theory, Observations and Techniques, ed. T. Rimmele, K. S. Balasubramaniam, \& R. Radick, Proc. 17th NSO/SP Summer Workshop, National Solar Observatory, Sunspot, New Mexico: ASP Conf. Ser., 183, 486

Wilson, P. R. 1962a, MNRAS, 123, 287

Wilson, P. R. 1962b, MNRAS, 124, 231

Wilson, P. R. 1962c, MNRAS, 124, 383

Wilson, P. R. 1963, MNRAS, 126, 393

Wilson, P. R. 1968, ApJ, 151, 1029 